International Journal of Bifurcation and Chaos, Vol. 10, No. 5 (2000) 959-970

(c) World Scientific Publishing Company

\title{
EXPERIMENTAL DEFINITION OF THE BASIN OF ATTRACTION FOR CHUA'S CIRCUIT
}

\author{
GUIDO PEGNA, RITA MARROCU, ROBERTO TONELLI and FRANCO MELONI \\ INFM - Dipartimento di Scienze Fisiche, Universitá di Cagliari, \\ Cittadella Universitaria di Monserrato, 09042 Monserrato (CA), Italy \\ GIOVANNI SANTOBONI \\ Centre for Nonlinear Dynamics and its Applications, \\ University College London, Gower Street, London WC1E 6BT, UK
}

Received July 15, 1998; Revised September 2, 1999

\begin{abstract}
In this paper we study the experimental determination of the basin of attraction for the Chua's circuit by means of an electronic device that is able to select initial voltages and to show the early stages of the subsequent trajectory on the oscilloscope. The results are shown and discussed in cases of multistability of periodic and chaotic solutions.
\end{abstract}

\section{Introduction}

Chaos is a dynamic phenomenon that can arise in physical nonlinear systems of very different natures, but nowhere else is it so ubiquitous and easily observed as in electronic circuits [Thompson \& Chua, 1995]. We can identify two main reasons for this. First, the simplicity and inexpensive nature of the devices used, and second, the physics of electronic devices is well understood, so that the modeling equations of motion are the best expression of the particular physical phenomenon. These circuits could be integrated into LSI chips if massive practical applications should emerge.

The earliest observations of chaos in electronic circuits were in forced nonlinear oscillators, like the sinusoidally excited neon bulb relaxation oscillator [van der Pol \& van der Mark, 1927] and the forced negative resistance oscillator [Ueda \& Akamatsu, 1980]. But among all electronic circuits, Chua's circuit [Madan, 1993] deserves a special place, for its simplicity and universality. Simple because it consists of all linear elements but one, a nonlinear resistor (Chua's diode) with a piecewise-linear $v-i$ characteristic. Universal because all characteristic features of chaotic motion (like period- doubling, intermittency, torus breakdown, etc.) have all been observed in this circuit [Madan, 1993].

The presence of noise and inherent experimental uncertainty in the definition of physical parameters make the task of an exact comparison between experimental and numerical results unfeasible. Although the overall scenario may be qualitatively comparable, particular thresholds, basin boundaries, bifurcations, may be quantitatively hard to locate. On the other hand, it is quite common for the behavior of a chaotic circuit to be affected by the perturbation introduced by the switching mechanism at instant $t=0$, when a rough switch-on takes place. This peculiarity forbids the reproducibility of a particular experimental situation, especially when a specific initial condition is required to be set up.

In this paper, we try to experimentally reproduce the basin boundaries for different multistable solutions of Chua's circuit. Our task will be to set the initial voltages in the circuit as we like, with good precision. In order to carry out this investigation, the whole chaotic circuit has been redesigned, as we will explain later. A similar approach, although with different components have been carried out by [Heagy et al., 1994]. 


\section{Chua's Circuit}

In Fig. 1 we show a schematic representation of a single Chua's circuit. The equations of motion representing the circuit are

$$
\left\{\begin{array}{l}
C_{1} \frac{d v_{1}}{d t}=\frac{1}{R}\left(v_{2}-v_{1}\right)-f\left(v_{1}\right) \\
C_{2} \frac{d v_{2}}{d t}=\frac{1}{R}\left(v_{1}-v_{2}\right)+i_{L} \\
L \frac{d i_{L}}{d t}=-v_{2}-R_{0} i_{L}
\end{array}\right.
$$

where $v_{1}, v_{2}$, and $i_{L}$ are, respectively, the voltages across capacitors $C_{1}$ and $C_{2}$, and the current flowing through inductor $L$. $R$ and $R_{0}$ are the resistance at the top of the circuit and the internal resistance of the inductor, respectively. In Eq. (1), $f\left(v_{1}\right)$ is the piecewise-linear function

$f\left(v_{1}\right)=G_{b} v_{1}+\frac{1}{2}\left(G_{a}-G_{b}\right)\left(\left|v_{1}+B_{p}\right|-\left|v_{1}-B_{p}\right|\right)$

where $B_{p}$ is the breakpoint voltage and $G_{a}$ and $G_{b}$ are the slopes of the linear parts beyond the breakpoints.

We characterized the circuit by measuring all its parameters, and we found the values reported in Table 1 . The experimentally determined $v-i$ characteristic of Chua's Diode is shown in Fig. 2, with the measured points represented by open circles. The solid line represents the numerical fit used to derive those values present in Table 1; the fitting operation shows us that the $v-i$ characteristic is
Table 1. Experimental values used in this paper. The additional resistances $R_{a}$ and $R_{b}$ described in Sec. 2 have values such that $R / R_{a}=0.09$ and $1 / R_{b}=0.05 \mathrm{mS}$.

\begin{tabular}{cc}
\hline Parameters & Experimental \\
\hline$C_{1}$ & $(10.03 \pm 0.02) \mathrm{nF}$ \\
$C_{2}$ & $(91.32 \pm 0.02) \mathrm{nF}$ \\
$L$ & $(19.50 \pm 0.05) \mathrm{mH}$ \\
$R_{L}$ & $(2.6000 \pm 0.0003) \Omega$ \\
$G_{a}$ & $(-0.725 \pm 0.005) \mathrm{mS}$ \\
$G_{b}^{+}$ & $(-0.370 \pm 0.005) \mathrm{mS}$ \\
$G_{b}^{-}$ & $(-0.371 \pm 0.005) \mathrm{mS}$ \\
$B_{p}^{+}$ & $(1.045 \pm 0.002) \mathrm{V}$ \\
$B_{p}^{-}$ & $(0.957 \pm 0.002) \mathrm{V}$ \\
\hline
\end{tabular}

slightly asymmetric (although in some parts within the uncertainty of the measurement), due to the possibility of slight differences between the positive and negative saturation voltages of the amplifiers used in our circuit. So, for parameters in the formula (2) we use $G_{b}^{+}, B_{p}^{+}$, or $G_{b}^{-}$and $B_{p}^{-}$, whether $v_{1}(t)$ is positive or negative. A better match between positive and negative slopes of the $v-i$ characteristic could likely be obtained by the use of better quality operational amplifiers, such as, for instance, model AD712 by Analog Devices.

With a fourth-order Runge-Kutta integration algorithm with adaptive stepsize [Press et al., 1992] we simulated the flow generated by Eqs. (1). For

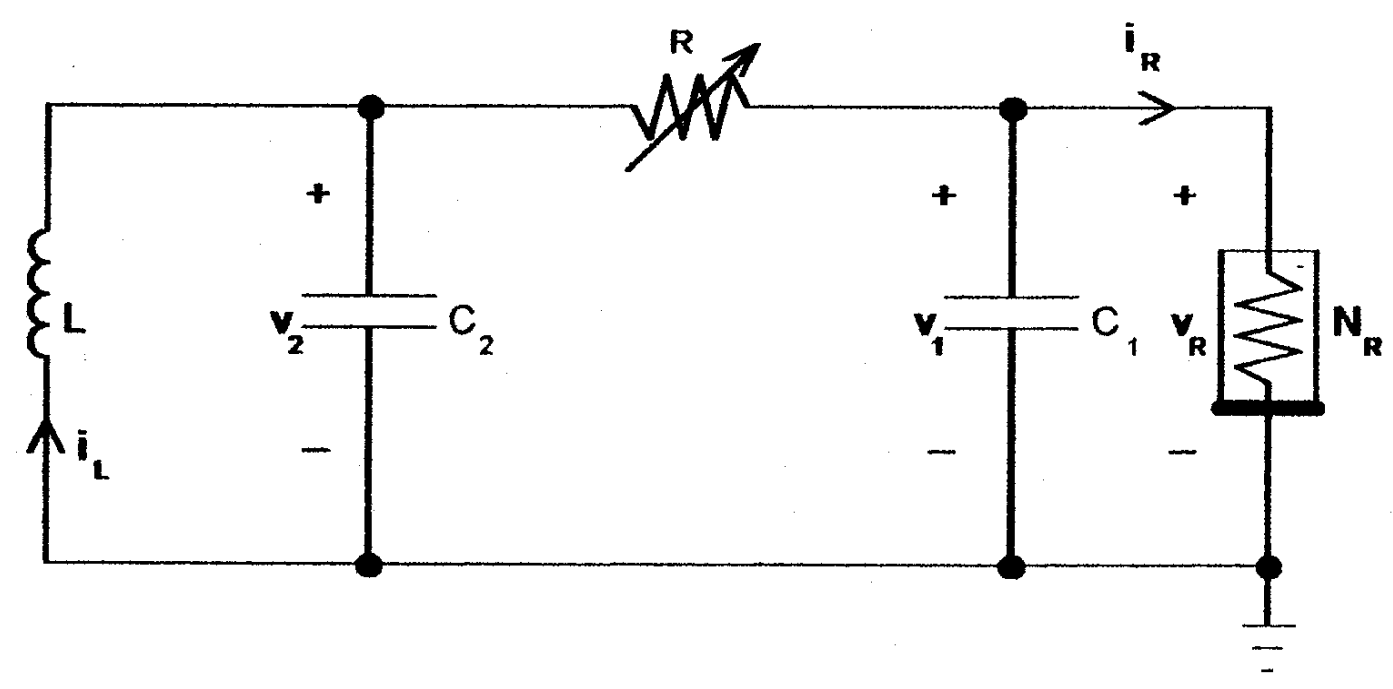

Fig. 1. Schematic representation of the Chua's oscillator. The Chua's oscillator consists of a linear inductor $L$ with a series internal resistance $R_{0}$, a linear resistor $R$, two capacitors $C_{1}$ and $C_{2}$, and a nonlinear resistor $N_{R}$. 


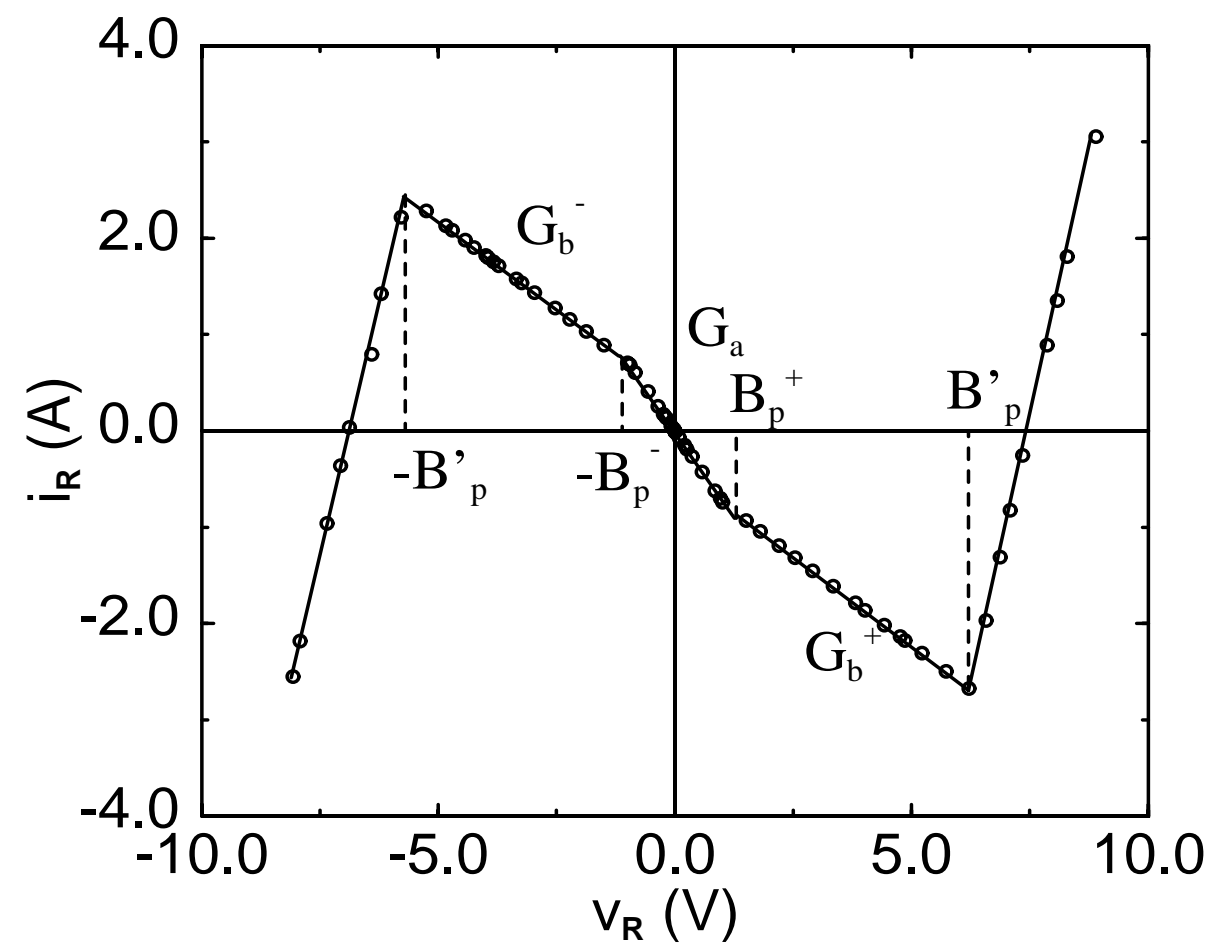

Fig. 2. Measured $v-i$ characteristic of the nonlinear resistor $N_{R}$. The circles represent the experimental points, while the solid line represents the numerical fit used to extract the parameter values. In the negative slope regions, the inner part has slope $G_{a}$, while for the outer part the slope is $G_{b}^{ \pm}$. For higher $\left|v_{R}\right|$-values the slope becomes positive, as for every physical realizable nonlinear resistor. The fitted values revealed that $G_{b}^{+} \neq G_{b}^{-}$and $B_{p}^{+} \neq B_{p}^{-}$, so that the characteristic is asymmetric.

the values reported in Table 1 the numerical solutions are slightly different from experimental ones. Our opinion is that the difference is generated by the presence of parasitic resistances in all the circuit set-up, but mainly by the hysteresis cycle of the inductor $L$, that we call $R_{a}$, variable in varying the resistance $R$ at the top of the circuit, and a fixed contribution from the two capacitors, whose effect is to change slightly the form of the $v-i$ characteristic (2). If the elaborated set-up carries with it an additional resistance $R_{b}$, the effect of connecting such a resistance in parallel with the negativeresistance diode is to change the slopes of the curve (2) as $G_{a} \rightarrow G_{a}+G$, and $G_{b} \rightarrow G_{b}+G$, where $G=1 / R_{b}$. On the other hand, the presence of the resistance $R_{a}$ modifies the Eqs. (1), as if there is a resistance in parallel with the capacitor $C_{2}$, so the second equation of the circuit should be modified as

$$
C_{2} \frac{d v_{2}}{d t}=\frac{1}{R}\left[v_{1}-v_{2}(1+\alpha)\right]+i_{L}
$$

where $\alpha=R / R_{a}$. The modified version of the equations of motion with the corrections $\alpha=0.09$, and $G=0.05 \mathrm{mS}$ gives a much better agreement with the numerical and the experimental behavior.
Figure 3 shows the bifurcation diagram of Chua's circuit, Eqs. (1), in the interval $R \in$ $[1.4,2.2] \mathrm{k} \Omega$. The different colors identify different coexisting solutions of Eqs. (1). The black points form the double-scroll chaotic attractor, ranging in the interval $R \in\left[R^{\prime}, R^{\prime \prime}\right]$. The double-scroll attractor coexists, for a large interval of $R$-values, with a large-amplitude periodic solution, which we label $\mathbf{C}^{s}$, represented with blue points in Fig. 3 . The double-scroll attractor loses its stability at $R^{\prime}$ with trajectories converging towards $\mathbf{C}^{s}$, and at $R^{\prime \prime}$ through crises [Ott, 1993] giving birth to two new chaotic solutions called single-scroll attractors. We label these attractors $\mathbf{A}^{ \pm}$, whether the attractor stays mostly in the positive $\left(\mathbf{A}^{+}\right)$or negative $\left(\mathbf{A}^{-}\right) v_{1}$ axis. In Fig. $3, \mathbf{A}^{+}$and $\mathbf{A}^{-}$are represented by red and yellow points, respectively. At point $R^{\prime \prime \prime}$ there is a bifurcation in which the largeamplitude periodic solution $\mathbf{C}^{s}$ loses its stability, giving way to one of the two periodic attractors $\mathbf{C}^{ \pm}$. As resistance $R$ increases, the periodic solutions experience a reverse period-doubling bifurcation sequence, until the two fixed points $\mathbf{P}^{ \pm}$gain stability along the way. In Fig. 3 two points are chosen on the horizontal axis. The experimentally measured 


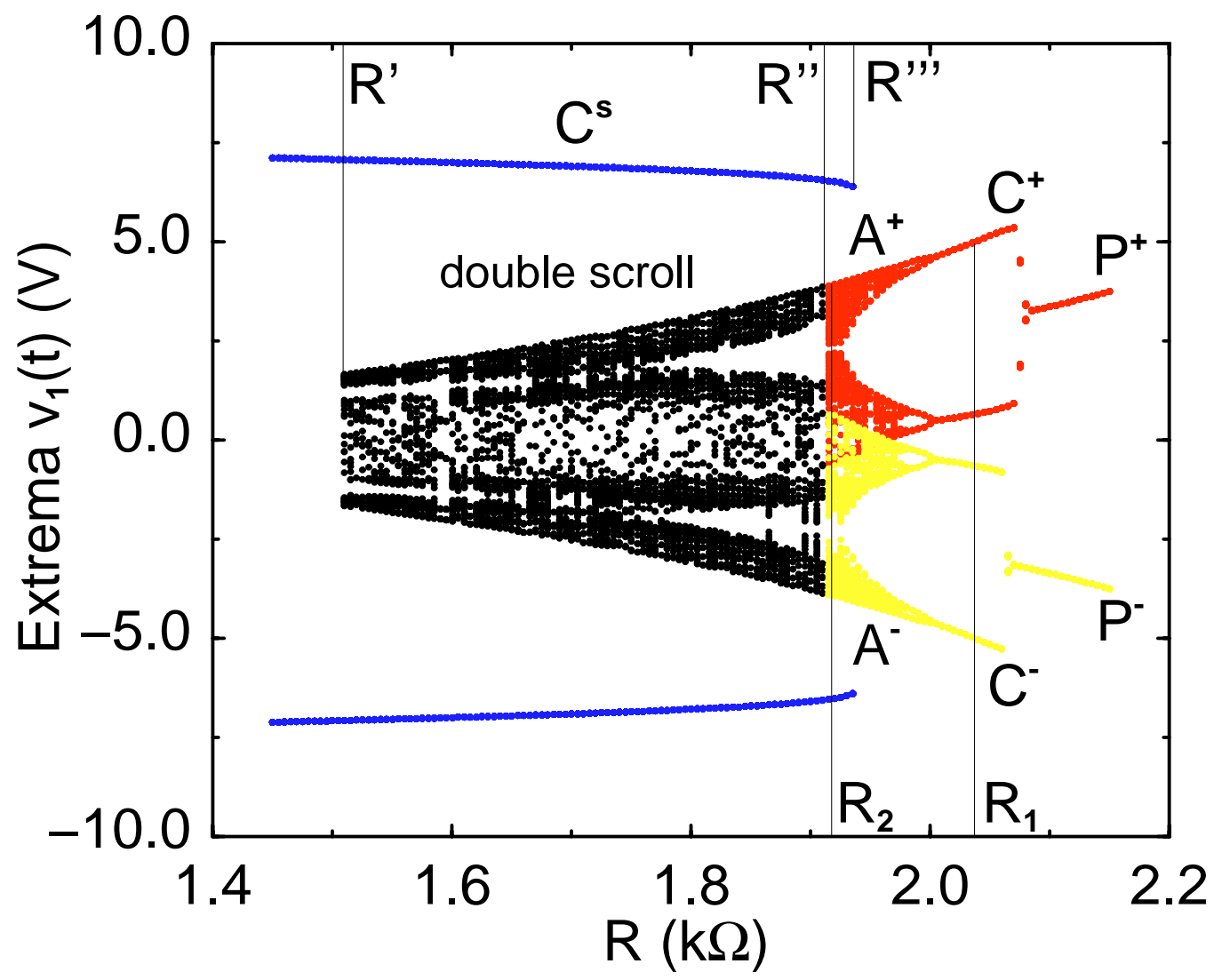

Fig. 3. Schematic bifurcation diagram of the Chua's circuit, Eqs. (1), obtained by sampling, for several values of $R \in$ $[1.4,2.2] \Omega$ the relative maxima and minima of the time history of $v_{1}(t)$. The different colors show all qualitatively different solutions. The black points belong to the double-scroll chaotic attractor, ranging, in the figure, in the interval $R \in\left[R^{\prime}, R^{\prime \prime}\right]$. In this range of $R$-values, the double-scroll attractor and the large periodic solution $\mathbf{C}^{s}$ (blue points) coexist. The red and yellow points belong, respectively, to $\mathbf{A}^{+}$and $\mathbf{A}^{-}$just after the crises in $R^{\prime \prime}$, so in the small interval $R \in\left[R^{\prime \prime}, R^{\prime \prime \prime}\right]$ the three solutions $\mathbf{A}^{ \pm}$and $\mathbf{C}^{s}$ coexist. Finally, the red and yellow branches give birth, reversing the period-doubling sequence in increasing $R$, to $\mathbf{C}^{ \pm}$and $\mathbf{P}^{ \pm}$.

value of $R_{2}=1.870 \mathrm{k} \Omega$, corresponds to a chaotic single-scroll solution just before (in decreasing $R$ ) the crises leading to the double-scroll solution, and $R_{1}=2.020 \mathrm{k} \Omega$, is located in the interval of stability of the $\mathbf{C}^{ \pm}$periodic solution. We will experimentally define and numerically compute the basins of attraction for these two values of the resistance $R$. For $R=R_{1}$ we have the multistablilty of $\mathbf{C}^{ \pm}$, while for $R=R_{2}$ the multistability of $\mathbf{A}^{ \pm}$and $\mathbf{C}^{s}$.

\section{Experimental Apparatus and Operations}

We now describe all the technical details of the system built to set the initial conditions to a precisely known value, and to show the first orbits in their free-running evolutions. In order to set initial conditions $v_{10}=v_{1}(0)$ and $v_{20}=v_{2}(0)$, the circuit must be put in a state, which we call SET, in which the following conditions are fulfilled:

(a) The two capacitors $C_{1}$ and $C_{2}$ are disconnected from the circuit.

(b) Chua's diode is set to a high impedence state (see Appendix A), to avoid any flow of current to the rest of the circuit.

(c) Generators of initial voltages $v_{10}$ across $C_{1}$ and $v_{20}$ across $C_{2}$, are connected to the respective capacitors.

(d) Inductor $L$ is disconnected from the circuit, to assure that the third initial condition is set to $i_{L}(0)=0$, as is usually done. Moreover, disconnecting $L$ when applying at point $H$ the initial voltage $v_{20}$ is a must in this circuit, owing to the high current which would otherwise flow into the low series resistance of the inductor, which would require an impractical high power voltage generator for $v_{20}$. 
Having accomplished the setting of initial conditions, steps (a)-(d) in reverse order must be executed in the shortest possible time to start the free-running dynamics of the chaotic circuit: disconnect $C_{1}$ and $C_{2}$ from the voltage generators and reconnect them to the circuit together with $L$; reset Chua's diode to its normal state. We call this second state RUN. For the observation of the first orbits with an analog oscilloscope the sequence of the alternate SET and RUN states is cyclically repeated at least fifty times per second to have a visible enough display. Moreover, the least possible time must be spent in the SET state, while the time in the RUN state must be variable to let observation time vary in order to display as much of the orbit as we like.

The complete diagram of the circuit is shown in Fig. 4 with related subfigures in boxes. At the top of Fig. 4 is shown the skeleton of the circuit, with relevant points $H, G, F$, and $D$. Figure 4 (top left) shows the circuits used to switch $C_{1}$ and $C_{2}$ from the SET to the RUN state, as explained below. Figure 4 (top-right box) reports our implementation of the Chua's diode (op-amps $D_{1}$ and $D_{2}$ ) [Kennedy, 1992] with its switching mechanism to a high-impedance state. In the bottom-left are sketched the two options available for inductor $L$ : The real and the synthetic one, together with their switching circuits. Finally, in the bottom-right box, there is a scheme of the command signals to all the analog switches.

A first tentative version of the circuit was based on reed relay as commutation devices. Unfortunately, in spite of its great simplicity, contact bouncing had a detrimental effect on the repeatability of trajectories. This fact led us to an entirely new design based on electronic commutators in order to have fast, clean and reproducible commutations.

The four analog switches contained in the 4066 CMOS integrated circuit have an ON resistance which is too high $(80-350 \Omega)$ and too dispersed, to be used as commutators in the insertion mechanism of initial voltages. For this reason, we introduced "ideal electronic switches" whose principle is sketched in Fig. 5.

In such a structure, the operational amplifier transforms the real analog switch inserted in its feedback loop into an ideal switch. When the switch is closed, the operational amplifier forces at its inverting input, which is the output of the circuit, a voltage identical to the voltage applied to its noninverting input, and it behaves as an ideal voltage generator. On the contrary, when the electronic analog switch is in the open state, if a FET input op-amp is used, the circuit is seen at its terminal as an infinite resistance.

As already reported in [Zhong \& Ayrom, 1985], we found that small changes in $R_{0}$ cause appreciable shifts in the period-doubling bifurcations, so, in order to give a more suitable definition of the value of $R_{0}$, we set this resistance to be the sum of two resistances in series, $R_{0}=R_{L}+R_{S}$, where $R_{L}$ is the internal resistance of inductor $L$ in Fig. 4 (bottomleft), and $R_{S}$ is variable.

With reference to the complete circuit diagrams in Fig. 4 (and to command signals shown in the bottom-right box), during the SET cycle operational amplifier $A 1$ and $A 2$ apply the inital voltages across $C_{1}$ and $C_{2}$ respectively, while $A 3$ and $A 4$ transform the values of resistances $R_{3}$ and $R_{6}$ in the Chua diode at infinity, causing point $D$ to be seen by the rest of the circuit as having infinite impedance. During the same cycle the ideal switch around op-amp $A 5$ is open, disconnecting from ground the lower terminal of inductor $L$. In such a configuration, during the SET state the initial voltages across the two capacitors are identical to $v_{10}$ and $v_{20}$, both within the $[-6,6] \mathrm{V}$ interval.

In our experimental configuration, $v_{10}$ and $v_{20}$ are displayed using high precision digital voltmeters. As shown in the bottom left box of Fig. 4, a synthetic inductor can be alternatively inserted in the circuit in place of the real one. It is built up by the circuit of op-amp $A 6$ (see Appendix B). Synthetic inductors are interesting because of specific advantages they have over real ones: Their series resistance is adjustable down to very low values, allowing very high quality factors $Q$ to be obtained thus introducing another control parameter on the behavior of the chaotic circuit, which might be of relevance in possible future studies on coupled circuits. Moreover, synthetic inductors do not suffer the effects of the hystheresis of the core, and are easily integrated in LSI. In our circuit the switching of the synthetic inductor is simply obtained by inserting one of the analog switches of the 4066 i.c. into its feedback loop. 

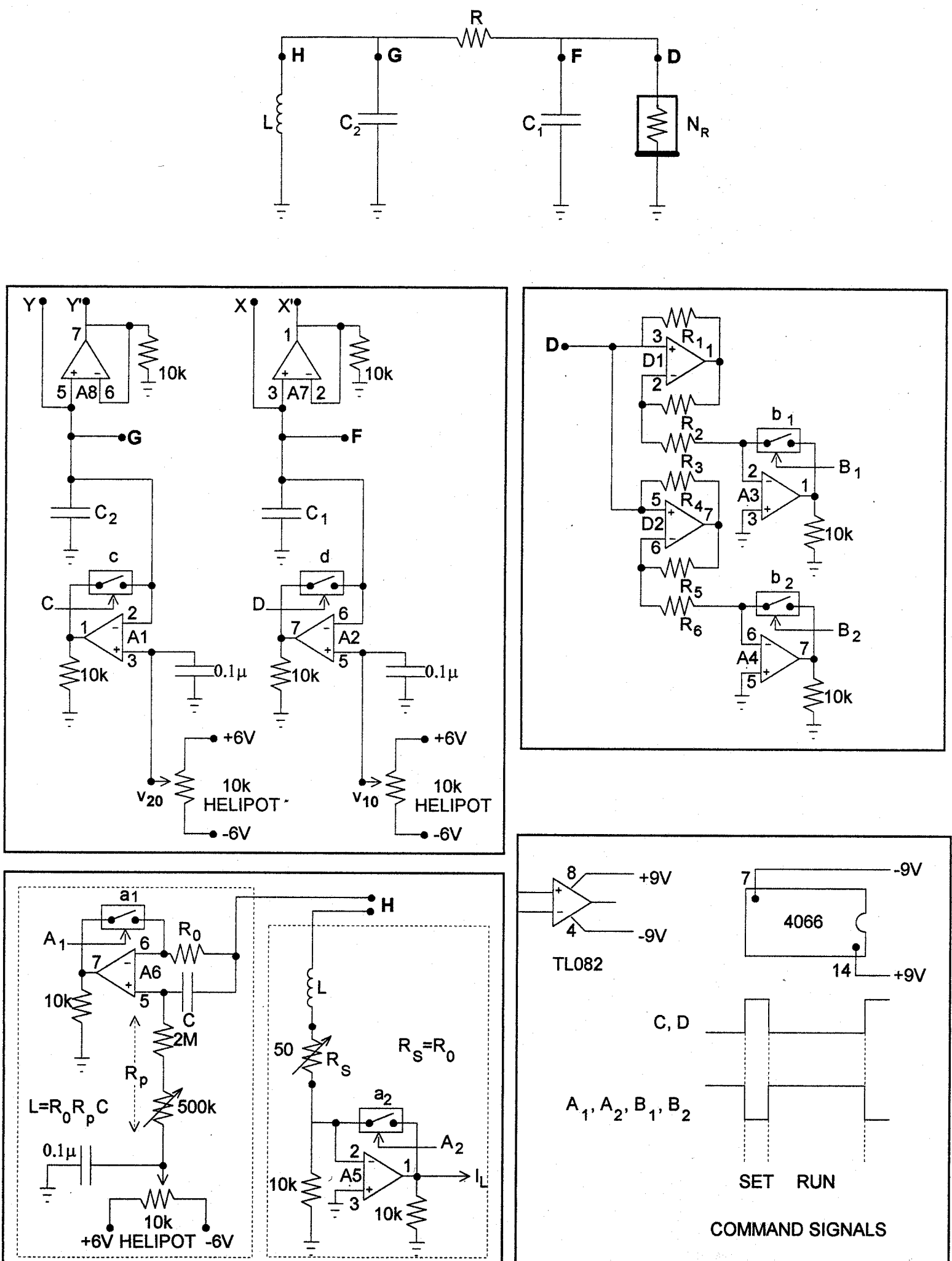

COMMAND SIGNALS

Fig. 4. The complete schematic diagram of the circuit. At the top of the figure is shown the skeleton of the circuit then, in the relative boxes are shown, the switching mechanism from the SET to the RUN state (top left), the structure of op-amps $D_{1}$ and $D_{2}$ (top right), the structure of inductor $L$ (bottom left), and the scheme of the command signals to all the analog switches (bottom right), respectively. The practical implementation uses two op-amps and six resistors to realize the Chua's diode. $R$ varies between $1.483 \mathrm{k} \Omega$ and $2.483 \mathrm{k} \Omega, R_{1}=R_{2}=218.6 \Omega, R_{3}=2.195 \mathrm{k} \Omega, R_{4}=21.79 \mathrm{k} \Omega, R_{5}=21.86 \mathrm{k} \Omega$, $R_{6}=3.28 \mathrm{k} \Omega$, in addition to the values previously reported in Table 1 . The relative uncertainty on the resistances $R_{1} \div R_{6}$ is $10^{-4}$. 


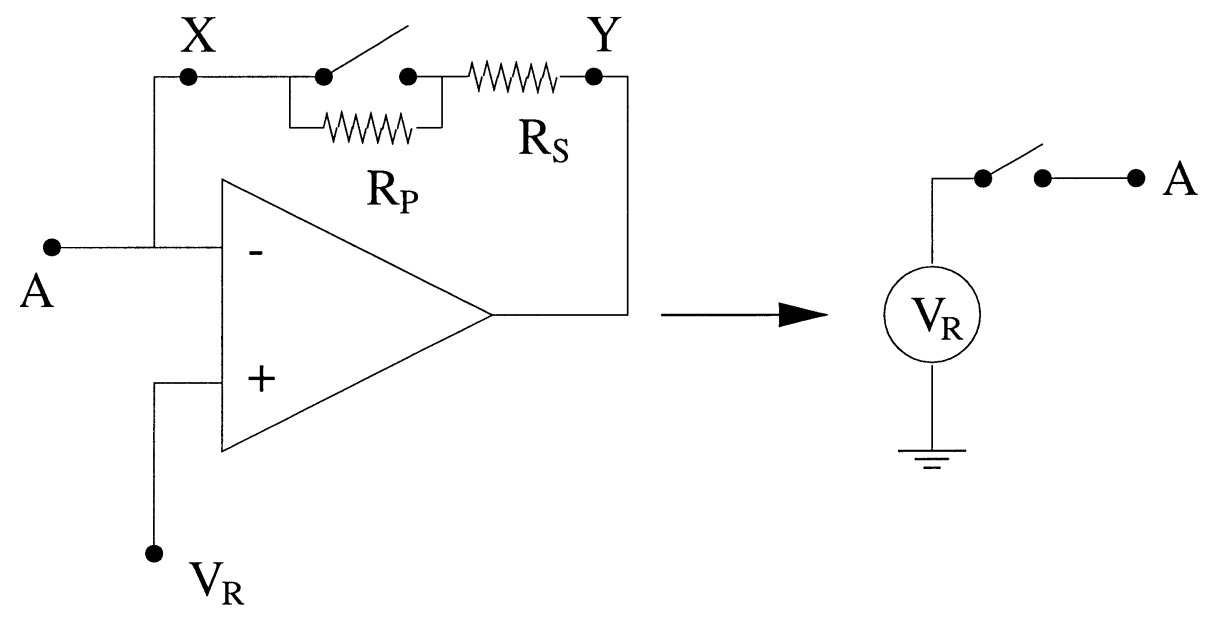

Fig. 5. Ideal switch mechanism.

The logic of the driving signals supplied to the integrated analog switches is as follows:

\begin{tabular}{l|c|c|c|c} 
& $a$ & $b$ & $c$ & $d$ \\
\hline SET & 0 & 0 & 1 & 1 \\
\hline RUN & 1 & 1 & 0 & 0
\end{tabular}

where $a$ and $b$ are all the switches indicated in the schematic diagrams of Fig. 4 with indices 1 and 2 . A suitable command circuit (not reported here) is built up on another board which supplies driving signals in true and complementary form and which allows the timing of the RUN state to be varied within large limits.

Output signals $v_{1}$ and $v_{2}$ (taken from the points $X$ and $Y$ ) are bufferized by op-amps $A 7$ and $A 8$, while points $X$ and $Y$ are directly accessible. Finally, for monitoring purposes, an additional signal proportional to $i_{L}$ is made available at the output of amplifier $A 5$.

\section{Experimental and Numerical Basin Boundaries}

We experimentally mapped two different basins of attraction for two different values of resistance $R$. Both cases will be compared with their numerical counterparts. The experimental basins were obtained by first selecting the edge point $\left(v_{10}, v_{20}\right)=$ $(-6,-6) \mathrm{V}$, still with $i_{L}(0)=0$. We then varied the value of $v_{20}$ continuously, observing the convergence of each trajectory, until we reached point $v_{20}=+6 \mathrm{~V}$.
After this, we repeat the procedure with an initial increment in the $v_{10}$ axis of $0.5 \mathrm{~V}$, but choosing smaller increments whenever the boundaries seem to be more irregular, until we arrive at the point where $\left(v_{10}, v_{20}\right)=(+6,+6) \mathrm{V}$. The crucial point is that the whole basin is determined "on the flight", i.e. observing the evolution of the orbits never switching off the circuit.

Following this procedure, we determined the basins of attraction for the two different values $R_{1}=2.020 \mathrm{k} \Omega$ (Fig. 6) and $R_{2}=1.870 \mathrm{k} \Omega$ (Fig. 8). In the periodic case, there are slight differences between experimental and numerical values. The peculiar characteristic of this kind of basin is that it is arranged by alternating stripes, but looking at Figs. 6 and 7, the number and width of these stripes are slightly different. In both figures the colors red/yellow define initial conditions converging towards $\mathbf{C}^{+} / \mathbf{C}^{-}$respectively. Now we turn to the chaotic case. Looking at the basin reproduced in Fig. 9, the outer blue zone marks all initial conditions whose convergence is towards the large periodic orbit, while the internal red/yellow zones with their peculiar boundaries represent convergence towards the single-scroll chaotic attractors $\mathbf{A}^{+} / \mathbf{A}^{-}$ pointing, respectively, in the positive or negative $v_{1^{-}}$ direction. Figure 9 has been produced with numerical integration of Eqs. (1). After 100000 integration steps, if the trajectory has a positive/negative $v_{1}$-value, the point is marked as belonging to the red/yellow basin.

For $R_{2}=1.870 \mathrm{k} \Omega$ (numerical) the resulting orbits $\mathbf{A}^{+}$and $\mathbf{A}^{-}$are chaotic single-scroll solutions, and from Fig. 3, that value of $R$ is closer to the crises giving rise to the chaotic double-scroll 


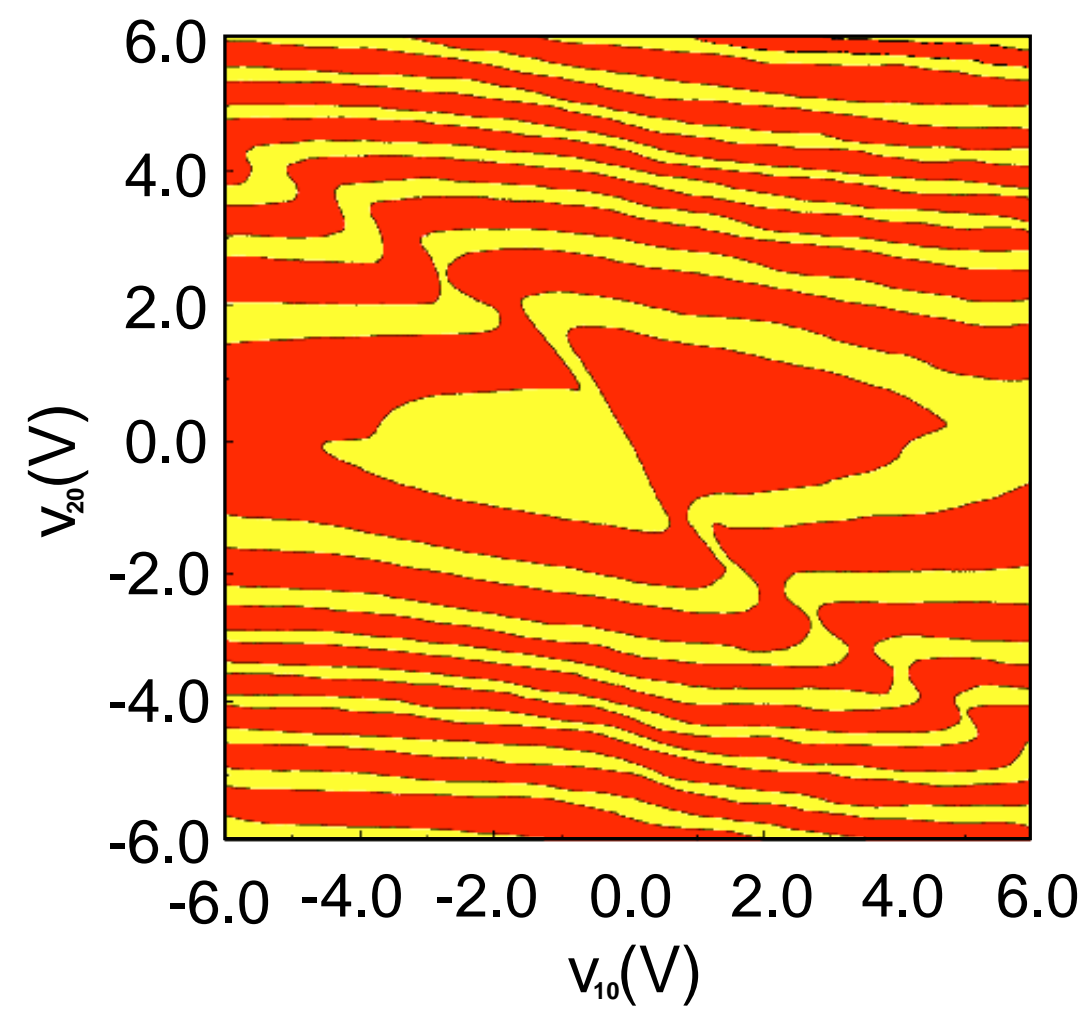

Fig. 6. Experimentally defined basin of attraction for $R=2.020 \mathrm{k} \Omega$. Initial conditions in the red zone converge towards the periodic solution $\mathbf{C}^{+}$, while initial conditions in the yellow zone converge to the solution $\mathbf{C}^{-}$. The comparison with the numerical result in Fig. 7 shows that qualitatively the agreement is good, but few differences can be recognized; this plot shows an evident asymmetry, e.g. the width of the stripes is bigger for the red ones, a peculiarity not seen in the numerical result.

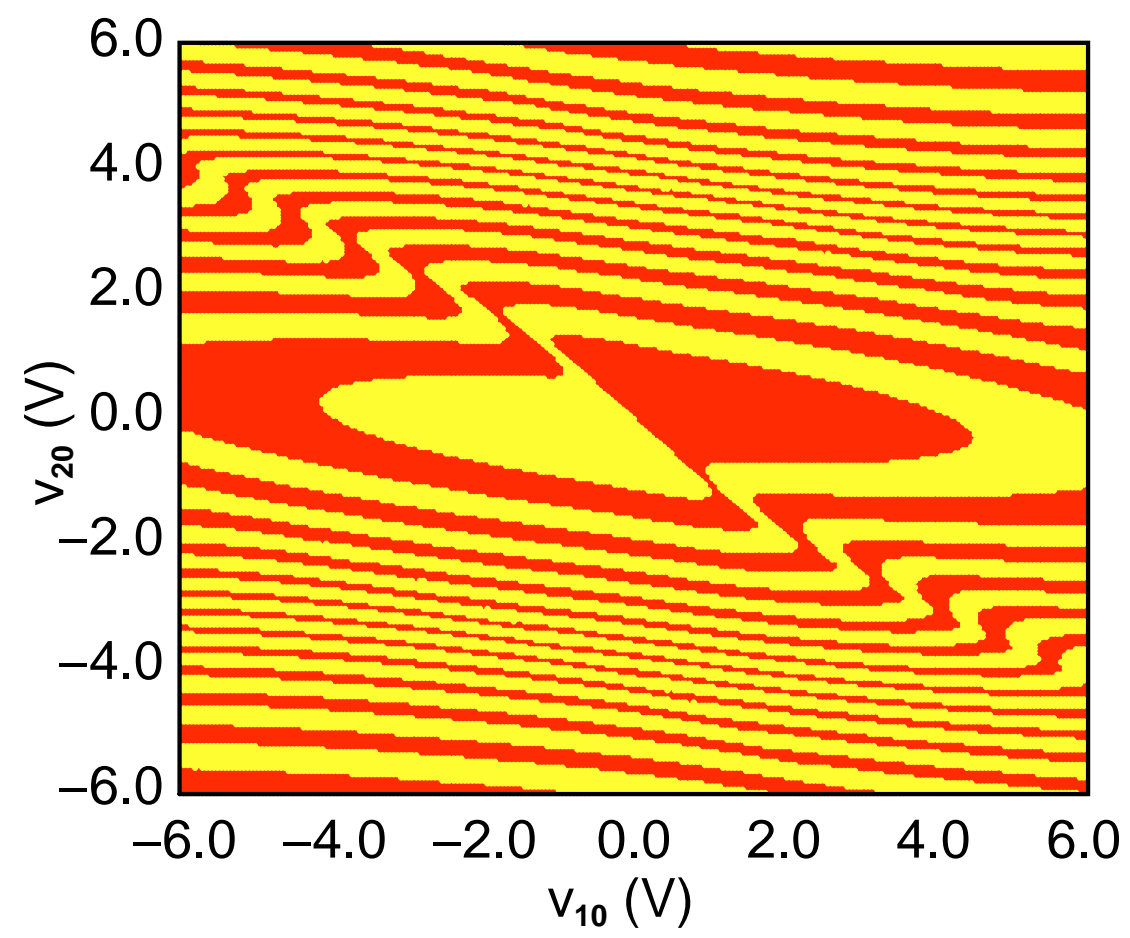

Fig. 7. Numerical basin of attraction for the convergence of Eqs. (1) onto the period-1 orbit $\mathbf{C}^{+}$or $\mathbf{C}^{-}$for $R=2.020 \mathrm{k} \Omega$. The meaning of the red and yellow zones is the same as in the previous figure. 


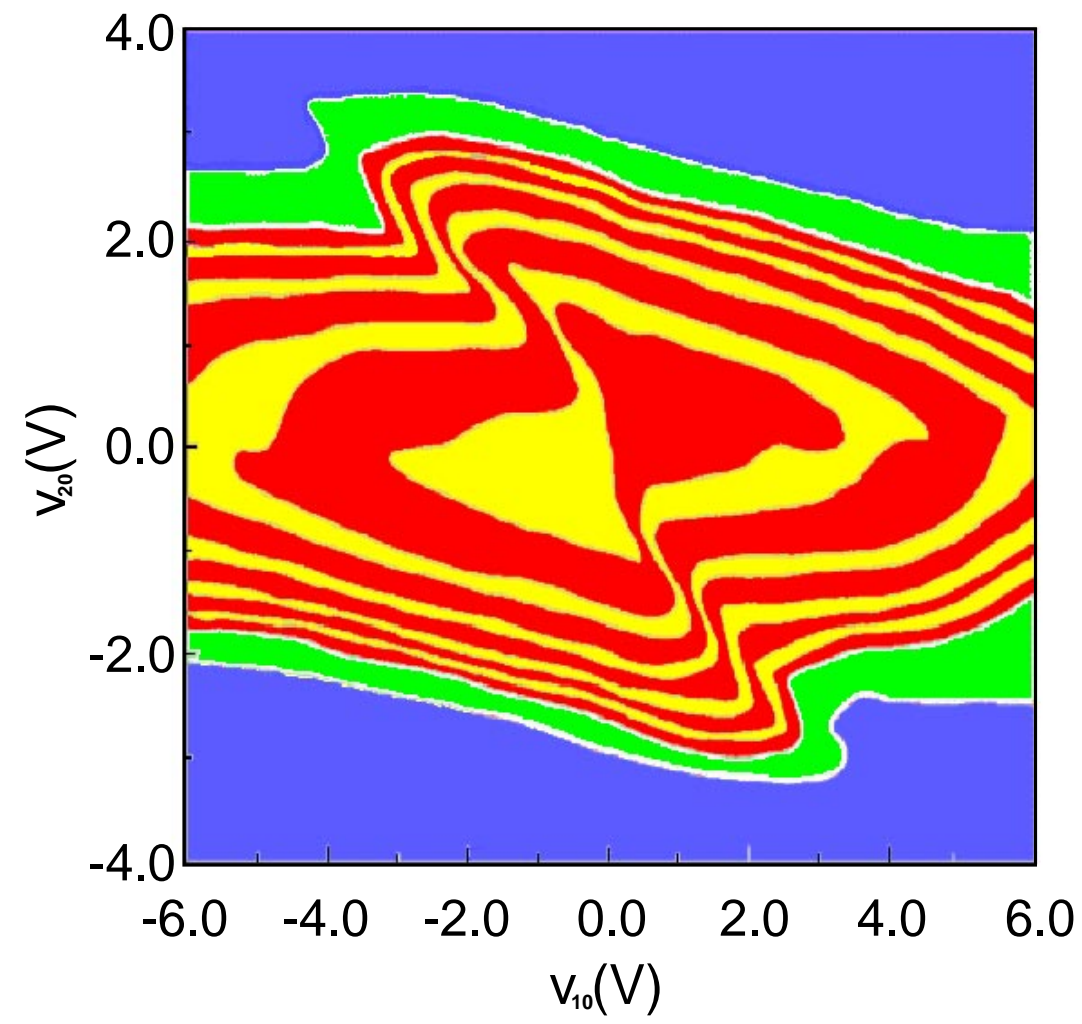

Fig. 8. Experimentally defined basin of attraction for $R=1.870 \mathrm{k} \Omega$. This value of $R$ is slightly bigger than $R^{\prime \prime}$ so, according to the bifurcation diagram in Fig. 3, the system is close to the transition from single-scroll to double-scroll solutions. Trajectories starting from the internal red and yellow zones converge respectively towards $\mathbf{A}^{+}$or $\mathbf{A}^{-}$, but two additional zones are present. The outermost blue zone indicates a part of the basin belonging to the large periodic solution $\mathbf{C}^{s}$ (as recognizable in Fig. 3 ) while the green zone in the middle indicates initial conditions which we have not been able to locate in either the red or yellow zone.

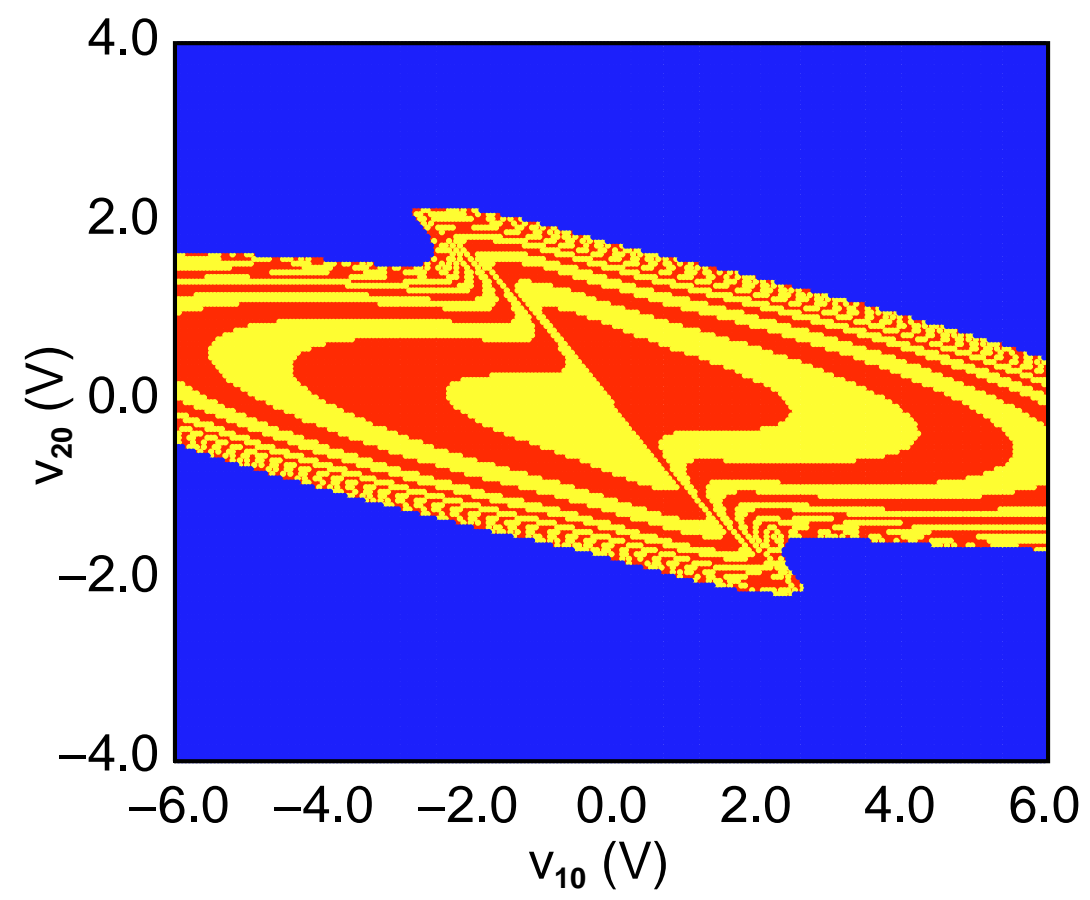

Fig. 9. Numerical basin of attraction for $R=1.870 \mathrm{k} \Omega$. The meaning of the red, yellow and blue zones is the same as in the experimental counterpart, Fig. 8. 
solution, so some intermittent behavior can be present, in which the trajectories wander across $\mathbf{A}^{+}$ and $\mathbf{A}^{-}$, before suddenly converging towards one of them.

Figure 8 represents the experimentally defined basin for the same value of the parameter. The outermost blue part represents the part of the basin converging towards the large external periodic solution $\mathbf{C}^{s}$, but between this part and the internal bistable part of the basin we have indicated in green an annular zone of the basin whose convergence we have not been able to assign to either $\mathbf{A}^{+}$or $\mathbf{A}^{-}$because of the intermittent behavior previously discussed.

\subsection{Role of the parameters}

The experimental and numerical results for this dynamical system show reasonable agreement, although we have to keep in mind the slight differences in the arrangment of the red/yellow strips in both basins. In this part of the paper we try to explain how differences in the circuit's parameters, even within the limits of experimental uncertainty, may change the shape of the basins.

First of all, we check the behavior of the basin of attraction on changing the value of resistance $R$, as in Fig. 1. As the value of $R$ is lowered, the perioddoubling sequence is passed through in the direction of the chaotic region, as can be seen in Fig. 3, and it also leads to a mixing of the two zones as seen in Figs. 6-9. So the red and yellow zones of the basin still equally share the available phase space (in the section $i_{L}(0)=0$ ), but they seem to be much more intertwined.

Regarding any deviation from the almost symmetric $v-i$ characteristic (2), its effect is to break the symmetry of the basins of attraction. For example, setting $\left|G_{b}^{+}\right|>\left|G_{b}^{-}\right|$the result is a significant enlargement of the basin for the $\mathbf{C}^{+}$, in correspondence to the larger $\left|G_{b}\right|$-value. Conversely, if the asymmetry is reversed, the visible effect is to enlarge the width of the yellow stripes. In conclusion, the steeper the slope of $G_{b}^{ \pm}$, the greater the portion of phase space occupied by $\mathbf{C}^{ \pm}$.

\section{Conclusions}

In this paper we described Chua's circuit with an electronic device capable of selecting initial voltages for it. We have detected a small disagreement between the experimental and the numerical behav- ior and we have proposed an improvement of the model which gives reasonable agreement. This device allowed us to map basins of attraction for multistable solutions, and the comparison between numerical and experimental results showed reasonable agreement.

We wish now to direct our next experiment towards a mapping of the initial conditions when the situation is far more critical and the systems are of higher dimensionality, i.e. when we deal with problems of synchronization of coupled circuits [Pecora \& Carroll, 1990]. In this case, the possibility of very complex basins of attraction must be taken into account [Ott \& Sommerer, 1994; Heagy et al., 1994], and some experimental evidences of riddled/intermingled behavior have appeared in recent scientific literature, using Chua's circuit [Kapitaniak, 1995; Kapitaniak et al., 1997].

\section{Acknowledgments}

The authors wish to thank P. Sirigu, A. Chessa and A. Bosin for their precious help in the practical and numerical realization of this paper. During the submission of the first version of the manuscript, the following paper, dealing with the same problem, appeared in the scientific literature [Virgin et al., 1998]. We acknowledge S. R. Bishop for pointing it out to our attention, and also for a critical reading of the manuscript. In particular, G. Sartoboni wishes to thank G. M. H. van der Heijden for many enjoyable discussions.

\section{References}

Bruton, L. T. [1969] "Network transfer functions using the concept of frequency-dependent negative resistance," IEEE Trans. Circuit Th. 16, 406-408.

Heagy, J. F., Carroll, T. L. \& Pecora, L. M. [1994] "Experimental and numerical evidence of riddled basins in coupled chaotic systems," Phys. Rev. Lett. 73, 3528-3531.

Kapitaniak, T. [1995] "Experimental observation of riddled behaviour," J. Phys. A28, 63-66.

Kapitaniak, T., Chua, L. O. \& Zhong, G.-Q. [1997] "Experimental evidence of intermingled basins of attraction in coupled Chua's circuit," Chaos Solit. Fract. 8, 1517-1522.

Kennedy, M. P. [1992] "Robust op amp realization of Chua's circuit," Frequenz 46, 66-80.

Madan, R. N. [1993] Chua's Circuit: A Paradigm for Chaos, ed. Madan, R. N. (World Scientific, Singapore). 
Ott, E. [1993] Chaos in Dynamical Systems (Cambridge Univ. Press).

Ott, E. \& Sommerer, J. C. [1994] "Blowout bifurcations: The occurrence of riddled basins and on-off intermittency," Phys. Lett. A188, 39-47.

Pecora, L. M. \& Carroll, T. L. [1990] "Synchronization in chaotic systems," Phys. Rev. Lett. 64, 821-824.

Press, W. H., Teukolsky, S. A., Vetterling, W. T. \& Flannery, B. P. [1992] Numerical Recipes in C (Cambridge Univ. Press).

Thompson, J. M. T. \& Chua, L. O. [1995] "Chaotic behaviour in electronic circuits," R. Soc. Phil. Trans. 353.

Ueda, Y. \& Akamatsu, N. [1980] "Chaotically transitional phenomena in the forced negative-resistance oscillators," IEEE Trans. Circuits Syst. 28, 217-224.

van der Pol, B. \& van der Mark, J. [1927] "Frequency demultiplication," Nature 120, 363-364.

Virgin, L. N., Todd, M. D., Begley, C. J., Trickey, S. T. \& Dowell, E. H. [1998] "Basins of attraction in experimental nonlinear oscillators," Int. J. Bifurcation and Chaos 8, 521-533.

Zhong, G.-Q. \& Ayrom, F. [1985] "Experimental confirmation of chaos from Chua's circuit," Int. J. Circuit Th. Appl. 13, 93-98.

\section{Appendix A}

The Chua's diode actually constitutes of the parallel of two negative conductance devices with suitable $v-i$ slopes and breakpoints. One such device is sketched in Fig. 10, together with its $v-i$ characteristic. Its conductance, as seen at point $D$, is given by $G=-\left(R_{2} / R_{1} R_{3}\right)$, so in our case $G=-1 / R_{3}$, since $R_{1}=R_{2}$. In order to set the device to a high-impedance state, one of the possibilities is to let $R_{3} \rightarrow \infty$, which is accomplished by means of perfect switches given by the op-amps $A 3$ and $A 4$ and real switches $b_{1}$ and $b_{2}$, respectively (see Fig. 4). Owing to the fact that at the instant when $R_{3}$ is disconnected, the initial voltge $v_{10}$ is simultaneously applied across $C_{1}$ (and hence at point $D$, Fig. 4), both inputs of the op-amp together with its output will be driven at the voltage $v_{10}$, no saturation of the op-amp occurring. The amplifier simply reacts forcing voltage $v_{10}$ at its inverting input. Being the noninverting input and the output of the opamp at the same voltage $v_{10}$, no current flows into $R_{1}$, as the impedance at point $D$ is actually seen as infinite.

\section{Appendix B}

The circuit shown for our synthetic inductor is likely to be a derivation of the so-called FDNR (Frequency Dependent Negative Resistor), a well assessed device introduced by Bruton [1969], widely used in the design of active filters. The version of the
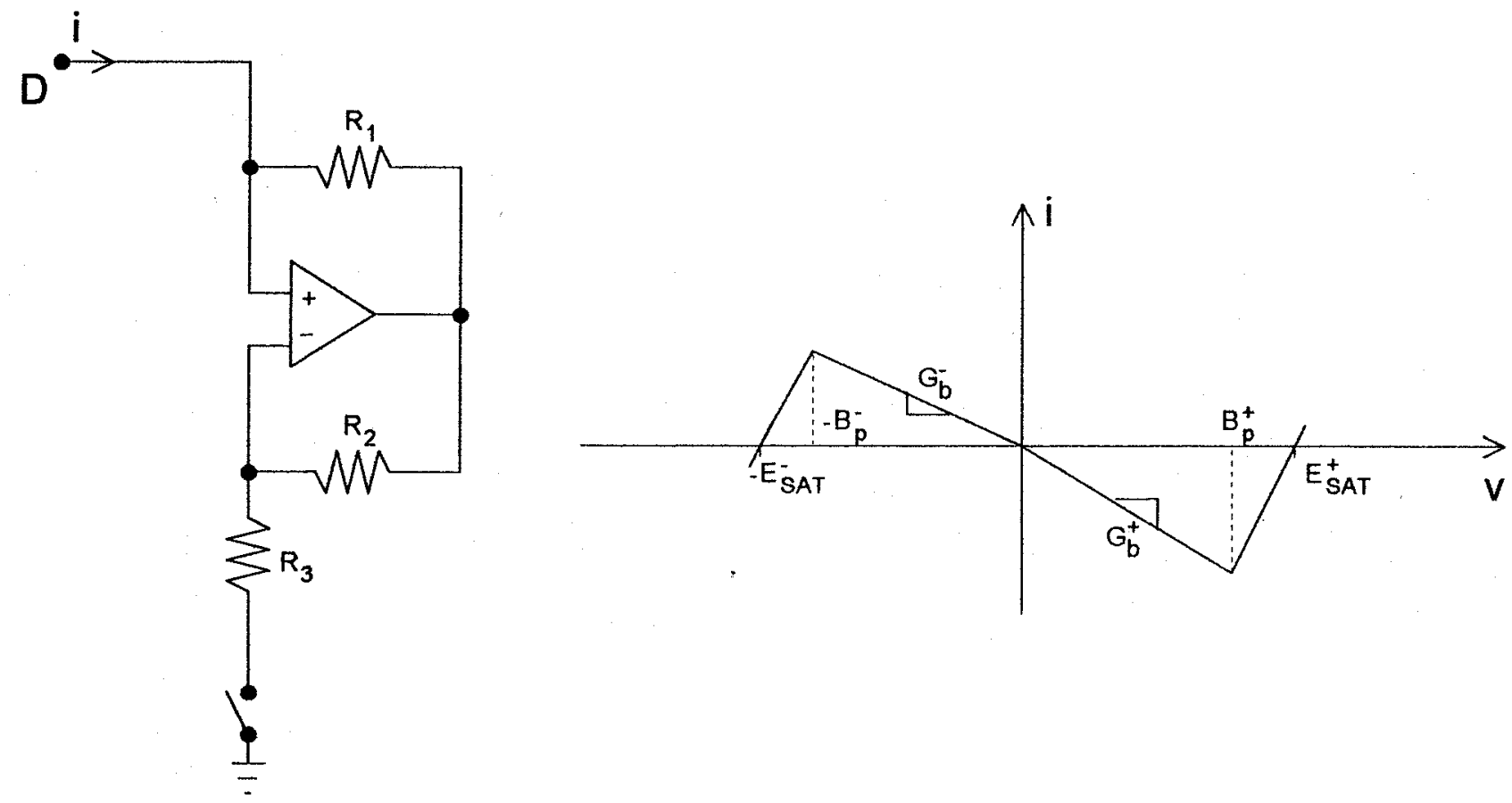

Fig. 10. The parallel of two negative conductance device constituting the Chua's diode and its $v-i$ characteristic. 

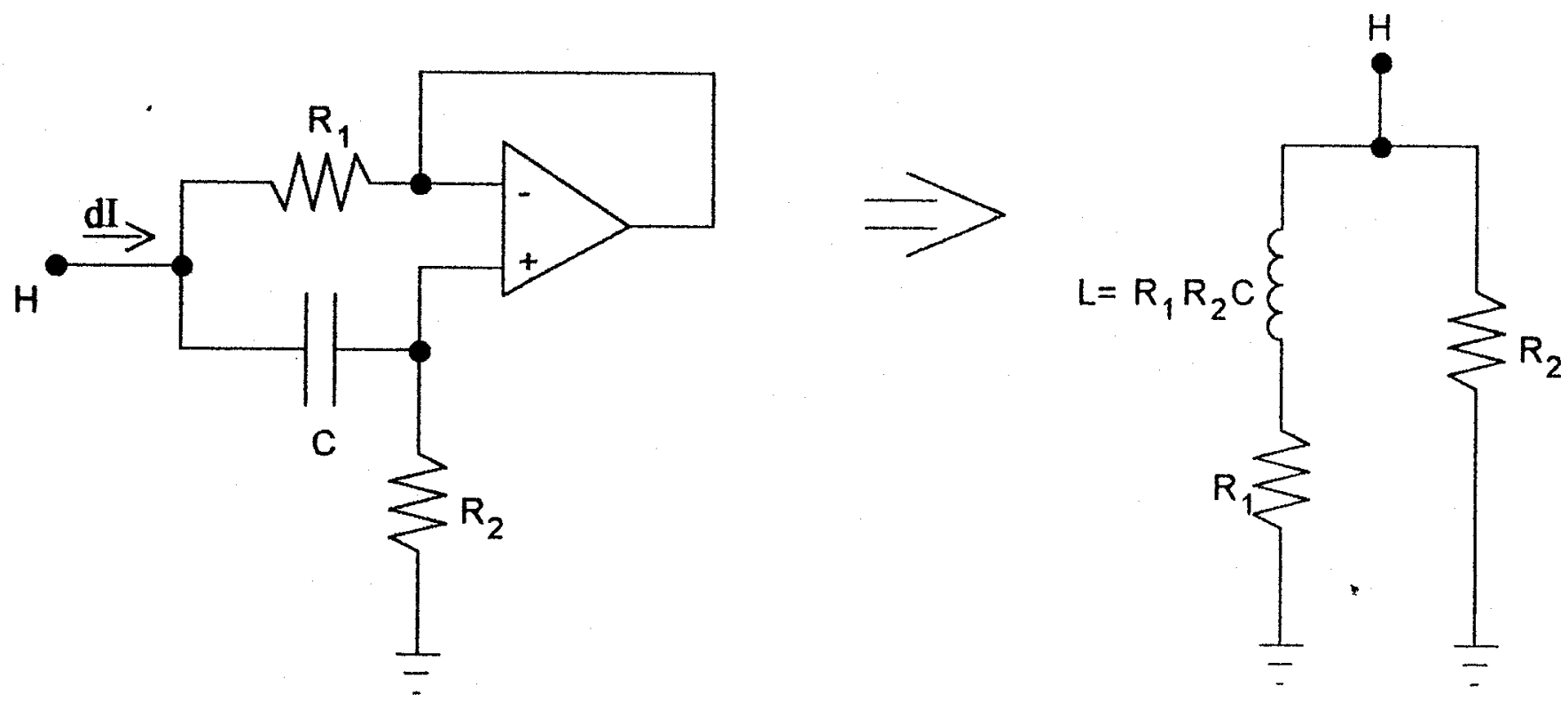

Fig. 11. Structure and working principle for the synthetic inductor.

circuit proposed here is exactly as reported in: Linear applications, Vol. 1, National Semiconductor Co., Santa Clara, Ca. 1973, p. AN31-15. A semiqualitative and somewhat approximate explanation of its working principle is as in Fig. 11.

At time $t=0$ imagine the start of a current variation $d I$ into point $H$. Point $B$ is at this time a virtual ground; therefore point $H$ will experience the voltage variation $d V=R_{1} d I$. This voltage variation will produce the current $I=C(d V / d t)$ in the capacitor and a finite voltage $V_{k}=R_{2} C(d V / d t)$ across $R_{2}$. The amplifier will react to this non- inverting input voltage by forcing and if the value of $R_{1}$ is small, approximately the same voltage will develop at point $H$. In conclusion, we have

$$
V=R_{1} R_{2} C \frac{d I}{d t}
$$

This functional relation between $V$ and $d I / d t$ shows that the circuit behaves as an inductor of value $L=R_{1} R_{2} C$. Actually, it behaves as a "nonperfect" inductor, its equivalent series resistance being $R_{1}$, while a parallel resistance $R_{2}$ appears connected across it. 